\title{
The Relationship between Thermoelectric and Thermodynamics Concepts and Dehumidifier for Understanding Physics in Senior High Schools
}

\author{
Abdul Rajak ${ }^{\mathrm{a}, 1}$, Ade Y. Nuryantini ${ }^{\mathrm{a}}$, M.Miftahul Munir ${ }^{\mathrm{b}, 2}$, and Khairurrijal ${ }^{\mathrm{a}, 3}$ \\ ${ }^{\text {a }}$ Physics of Electronic Materials Research Division, \\ ${ }^{\mathrm{b}}$ Theoretical High Energy Physics and Instrumentation Research Division, \\ Faculty of Mathematics and Natural Sciences, Institut Teknologi Bandung \\ Jalan Ganesa 10, Bandung 40132, Indonesia \\ 1 rajakphysics89@gmail.com, ${ }^{2}$ miftah@fi.itb.ac.id, ${ }^{3}$ krijal@ fi.itb.ac.id
}

\begin{abstract}
Today, the development of technology has reached at an amazing level. Either in households or industries, technology becomes an important necessity that facilitates human life. There are many technologies around us that use physics principles learned at senior high schools. However, many senior high school students are not aware that their daily life technologies are closely linked with the principles. The students only memorize physics formulas without knowing the theories and their applications. This paper describes the working principle of a dehumidifier, which is an equipment to make our body comfort by reducing room's humidity, related to the topic on thermoelectric given to senior high school students. The main working principle of dehumidifier is to change humid air into water droplets using a small fan and a cooling coil. The dehumidifier sucks humid, hot air by employing the small fan. The humid, hot air touches the cooling coil so that the water content in air thickens and it becomes water droplets. The water droplets finally drip to the collecting bucket. This principle is closely related to the concept of thermoelectric and thermodynamic concepts learned in high schools.
\end{abstract}

Index Terms: Dehumidifier, thermoelectric concept, Peltier effect, thermodynamics.

\section{Introduction}

Atmosphere always contains water vapor, which is a gas that is odorless, invisible and colorless. Water vapor has the same molecule structure with water but it is in the form and the state of gas. Water vapor is a result of evaporation of liquid water. Since evaporation is the change of water from a liquid state to a gaseous state, certain amount of heat is needed in evaporation. The concentration of water vapor in the atmosphere is called as humidity, which always changes depending on the local air temperature.

The concentration of water vapor can be expressed in absolute humidity, specific humidity, or relative humidity $[1,2]$. The total mass of water vapor per unit volume of air is referred to absolute humidity. The comparison between the mass of water vapor to the mass of moist air in a certain unit volume of air is called as specific humidity. Moist air mass is the total mass of all atmospheric gases contained while the relative humidity $(\mathrm{RH})$ is the percentage ratio between the pressure of water vapor to the pressure of saturated water vapor, as expressed in Eq. (1) [1].

$$
R H=\frac{P_{\mathrm{H}_{2} \mathrm{O}}}{P_{\mathrm{H}_{2} \mathrm{O}}^{*}(\text { sat })} \times 100 \%
$$

Humidity of air is also known as air wetness level. In some cases, very high levels of humidity cause adverse effects because high humidity will trigger the growth and reproduction of harmful bacteria more quickly [2]. Therefore, the equipment that is able to reduce humidity level become normal is needed. The equipment that can perform this function is called as dehumidifier whereas an instrument used to measure the humidity level is called as hygrometer.

Dehumidifier is technological equipment that serves to regulate the humidity of a room. It converts water vapor into water droplets through a system based on thermoelectric concept. The working principle of this equipment is to change humid air become water droplets by using a cooling coil and a small fan. Then, water droplets fall into the container vessel called as collecting bucket. The dehumidifier sucks heat from the humid air and then release the cool air [3].

In education, the introduction of scientific insight is needed in order to increase the motivation of learners in the study of science particularly physics. As we know physics is an important subject in the development of science and technology. In order to increase knowledge and insight for students and teachers on a variety of technology applications around us, this paper discusses detailed thermoelectric concepts which are applied in the dehumidifier technology as described above. This paper also reports simple experimental results on the performance of dehumidifier in reducing air humidity.

\section{Theory}

The concept of thermoelectric is to change heat energy of the temperature gradient into electrical energy or vice versa. Many thermoelectric applications are currently used, such as the use of heat differences in seabed and land to produce electrical energy. In general, there are 3 thermoelectric phenomena, namely: Seebeck, Peltier, and Thomson effects [4]. This paper discusses the Peltier effect because it is related to the concepts used in the dehumidifier technology.

The Peltier effect was first discovered by French physicist, Jean Charles Peltier in 1834. He observed a phenomenon that a temperature difference is generated after a flow of electric current in a semiconductor material. The 
occurrence of this temperature difference can be used to drain the amount of heat energy for a particular application (Figure 1). The generated heat flow is proportional to the electric current flowed as expressed in the following equation $[5,6]$ :

$$
Q=\Pi \cdot I
$$

where $\Pi$ is the Peltier coefficient of a semiconductor material used and $I$ is the current flowed. Some commonly used semiconductor materials are $\mathrm{Bi}_{2} \mathrm{Te}_{3}, \mathrm{PbTe}$ and $\mathrm{SiGe}$ [6].

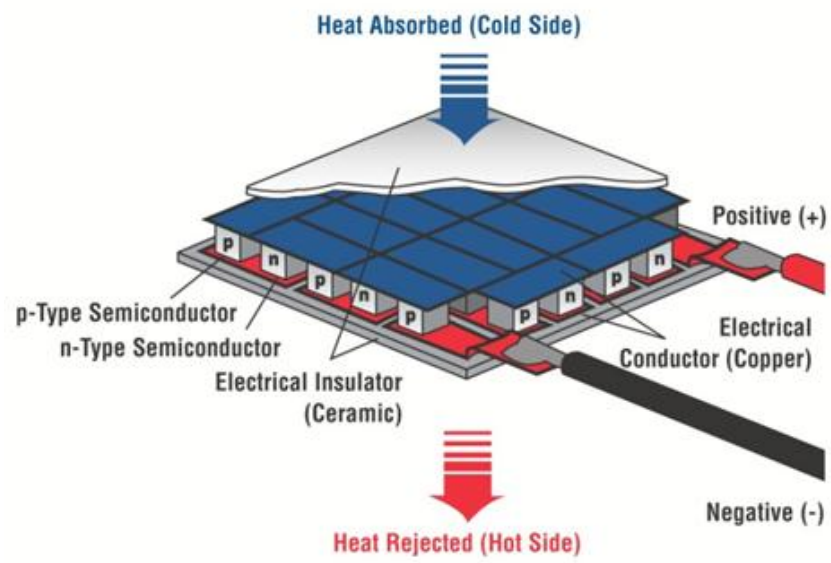

Fig.1 Scheme of heat flow on the Peltier effect [7]

The difference in temperature due to the Peltier effect causes an amount of heat flows from a high temperature reservoir to lower one. Temperature increases linearly with the amount of heat flow. Astrain, et al. [6] have modeled the linear relationship as shown in Figure 2.

Dehumidifier is one of the technological products based on the Peltier effect. This equipment is able to decrease high humidity of air becomes normal humidity through the process called as dehumidification by reducing the concentration of water vapor content in the atmosphere. There are several parameters that affect the time taken for dehumidification process, that is: air temperature, relative humidity, air flow velocity, and heat [2].

Dehumidifier is commonly used to protect the stock of medicines in pharmaceutical industries, to protect sensitive equipment in hospitals, and to maintain the level of humidity in the production areas. For these needs, the optimal relative humidity range is between 30 to $35 \%$.

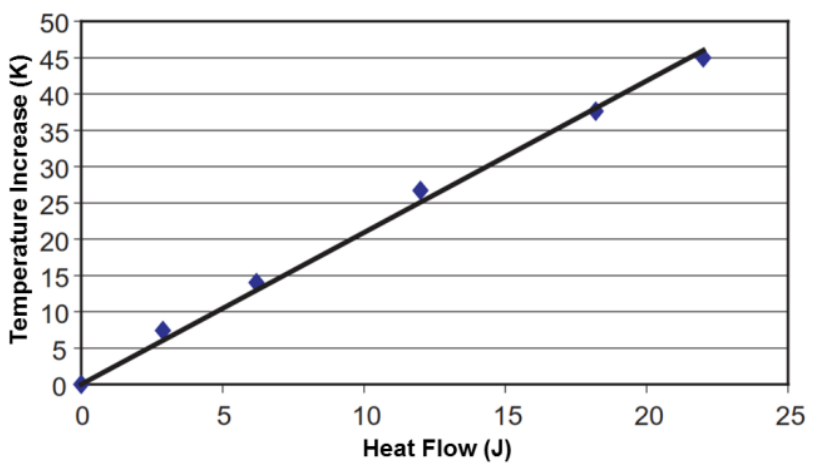

Fig.2 The relationship between heat flow and temperature increase [6]
Basically, the main working principle of dehumidifier is to absorb heat from the moist environment so that the process of condensation of water vapor occurs. The condensation process causes a phase change of water vapor into water droplets and the water droplets are collected in a tank located in the dehumidifier. A hydro sensor in the dehumidifier will respond if the tank is already full. The absorbed heat will then be released back to the other side of the dehumidifier. This cycle runs continuously until the relative humidity reaches a saturation state [3,8]. The complete diagram of the dehumidifier can be seen in Figure 3 .

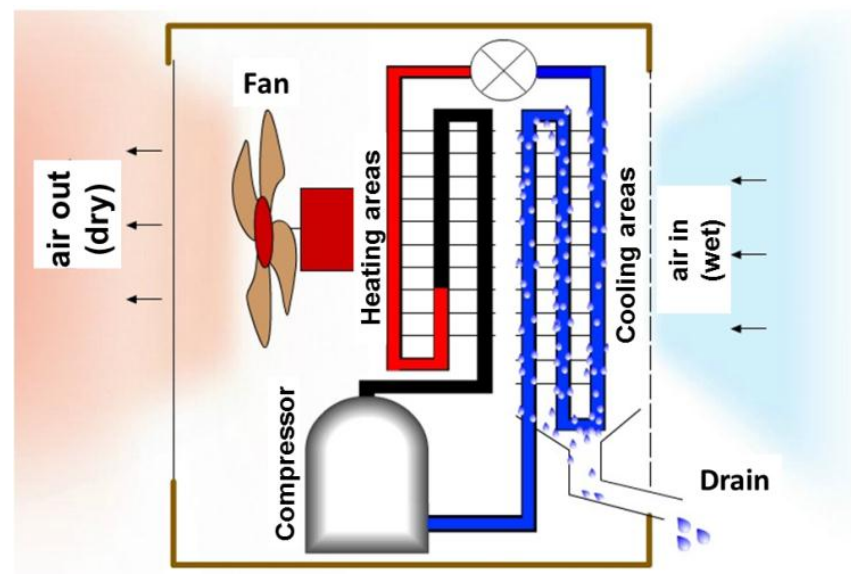

Fig.3 Diagram of dehumidification process [8]

There are two important concepts of physics that is closely related to the working principle of dehumidifier. The first concept is the phase change of water from vapor to liquid form and the second concept is the second law of thermodynamics stating that to flow heat from the lowtemperature reservoir to high-temperature reservoir an amount of work is needed as illustrated in Figure 4. These concepts are already learned in high school but an explanation of the applications of the concepts is still lacking.

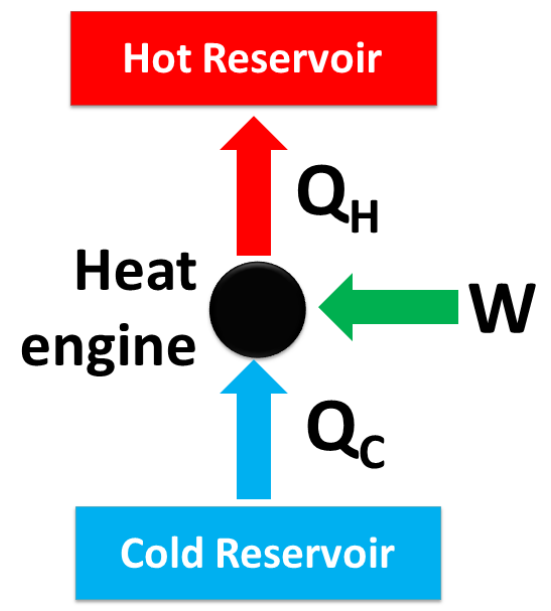

Fig.4 The second law of thermodynamics

Noting the working principle of dehumidifier, it is categorized as a heat engine. The moist environment is 
defined as the low-temperature reservoir, while the hightemperature reservoir is the environment that has low humidity (after processed by the dehumidifier). According to the second law of thermodynamic, the work $W$ exerted by the dehumidifier to flow heat from the cold reservoir to the hot reservoir is expressed by Eq. (3) [9].

$$
W=Q_{H}-Q_{C} ; Q_{H}>Q_{C}
$$

where $Q_{H}$ and $Q_{L}$ are the heat given to the hot reservoir and that released by the cold reservoir, respectively.

Hence, the coefficient of performance of a dehumidifier is expressed as

$$
C_{P}=\frac{Q_{C}}{W}=\frac{Q_{C}}{Q_{H}-Q_{C}}
$$

Because of heat and temperature are proportional, Eq. (4) can be rewritten as:

$$
C_{P}=\frac{T_{C}}{T_{H}-T_{C}}
$$

Other quantities which are very important in this case are the rate of heat absorption $(H)$ and power $(P)$, where $H=$ $Q / \mathrm{t}$ and $P=W / \mathrm{t}$ with $\mathrm{t}$ is time. Thus the coefficient of performance of a dehumidifier can also be expressed by [9].

$$
C_{P}=\frac{Q_{C}}{W}=\frac{H}{P}
$$

\section{Experimental}

We have conducted a simple experiment in order to obtain the relationship between size of the room and the rate of reducing relative humidity. The decrease of humidity was measured by a digital hygrometer that can display the percentage of relative humidity and ambient temperature (Figure 5.(b)). Measurements were taken at two different boxes with dimensions $(30 \times 22 \times 30) \mathrm{cm}$ and $(40 \times 25 \times 30)$ $\mathrm{cm}$. A mini dehumidifier purchased from Ace Hardware Indonesia was used in the experiment (Figure 5.(b)).

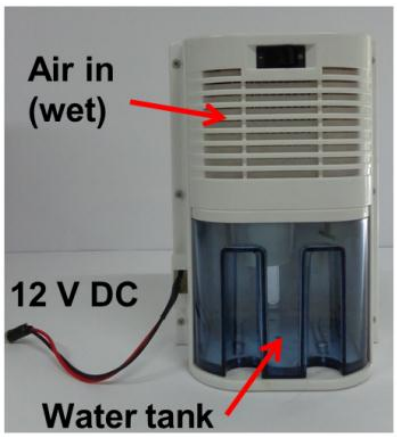

(a)

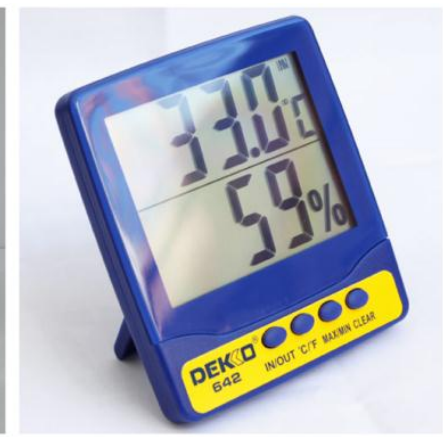

(b)
Fig.5. (a) Dehumidifier and (b) hygrometer

Both of the boxes were prepared at the same initial relative humidity. The hygrometers were placed in the boxes and the dehumidifiers were turned on simultaneously. We recorded the percentage of relative humidity and temperature measured by the hygrometer every 15 minutes for 150 minutes.

\section{Results and Discussion}

From the simple experiment, we obtained the humidity every 15 minutes for 150 minutes for each box as shown in Figure 6 . The temperature every 15 minutes for 150 minutes measurements are given in Figure 7.

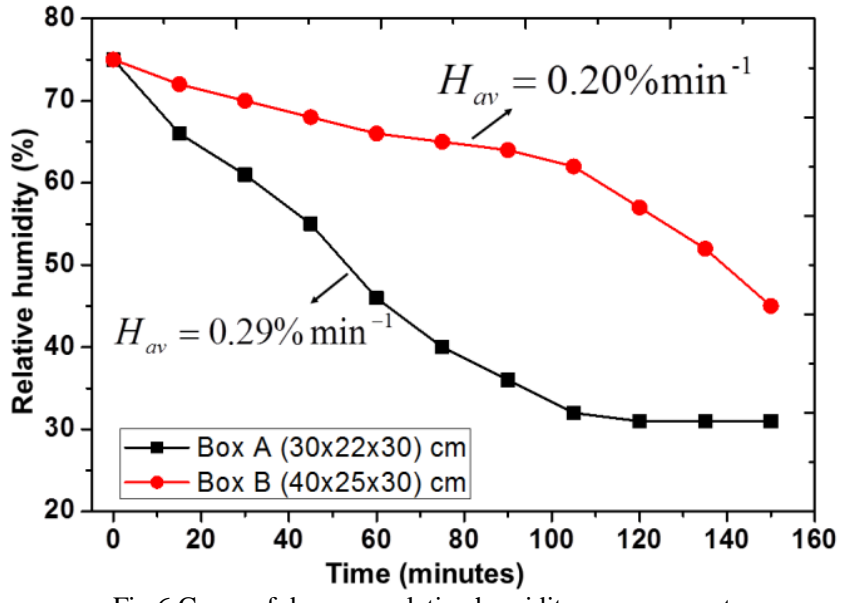

Fig.6 Curve of decrease relative humidity measurement

As seen in Figure 6, the initial of relative humidity in both boxes was set at $75 \%$. It was obtained that the small box has greater rate of decrease in the relative humidity $(0.29 \% / \mathrm{min}$.) than the big box does $(0.20 \% / \mathrm{min}$.). This happens because the total concentration of water vapor molecules in the small box is less than that in the big box.

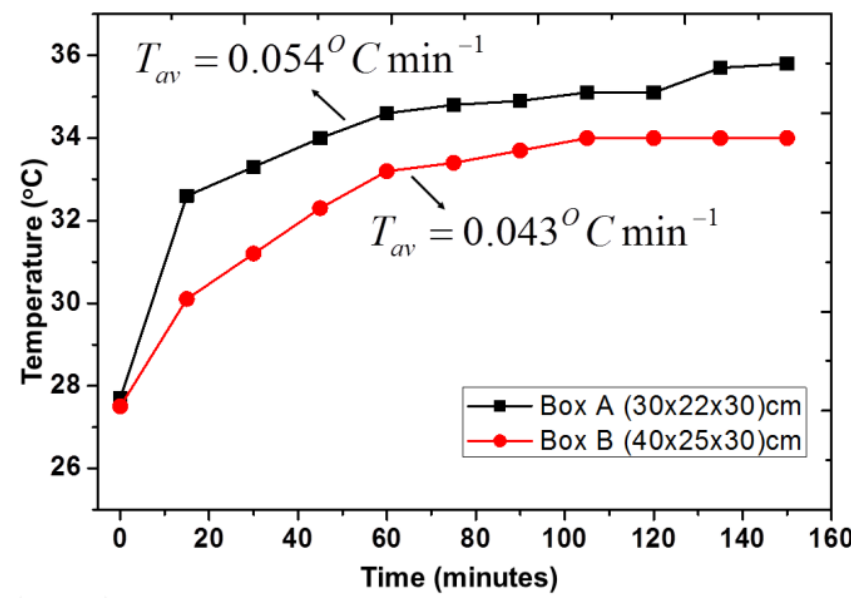

Fig.7 Curve of relationship between temperature increase and time taken

Figure 7 shows that the increase of temperature occurs faster in the small box $\left(0.054{ }^{\circ} \mathrm{C} / \mathrm{min}\right.$. $)$ compared to that in the big box $\left(0.043{ }^{\circ} \mathrm{C} / \mathrm{min}\right.$.). It means that the amount of heat needed in the small box to decrease the humidity is less than that in the big box. The dehumidifier will therefore demand more work for decreasing the humidity in the big box. 


\section{Conclusion}

We have described in detail the concept of thermoelectric and Peltier effect to explain the working principle of a dehumidifier in reducing the relative humidity of air in a room. The working principle of dehumidifier is based on the second law of thermodynamics in which the dehumidifier absorbs heat from a low-temperature reservoir and releases it into a high-temperature reservoir.

A simple experiment was performed using two boxes that have different sizes. It was obtained the decrease rate of humidity in the small box is higher than that in the big box. In addition, the small box has an increase rate of temperature higher than that in the big box.

\section{Acknowledgement}

This work was financially supported by the Community Services Grant, Institut Teknologi Bandung, in the fiscal year 2014

\section{References}

[1] N. David, K. Von Schilling, P. M. Wild, and N. Djilali., "In situ measurement of relative humidity in a PEM fuel cell using fibre bragg grating sensors," Int. J. Hydrogen Energ., vol. XXX, pp. 1-7, 2014 .

[2] N. Barroca, L. M. Borges, F. J. Velez, F. Monteiro, M. Gorski, and J. Castro-Gomes, "Wireless sensor networks for temperature and humidity monitoring within concrete structures," Constr. Build. Mater, vol. 40, pp. 1156-1166, 2013.

[3] X. H. Liu, X. M. Chang, J. J. Xia, and Y. Jiang, "Performance analysis on the internally cooled dehumidifier using liquid desiccant," Build. Environ., vol. 44, pp. 299-308, 2009.

[4] C. Goupil, W. Seifert, K. Zabrocki, E. Muller, and G. J. Snyder, "Thermodynamics of thermoelectric phenomena and applications," Entropy, vol. 13, pp. 1481-1517, 2011.

[5] M. Zebarjadi, K. Esfarjani, and A. Shakouri., "Nonlinear Peltier effect in semiconductors," Appl. Phys. Lett., vol. 91, pp. 122104, 2007.

[6] D. Astrain, J. G. Vian, and J. Albizua., "Computational model for refrigerators based on Peltier effect application,” Appl. Therm. Eng., vol. 25, pp. 3149-3162, 2005.

[7] http://www.delta-fan.com

[8] I. Asmawi and M. Shofyan., "Modifikasi split air conditioning sebagai unit dehumidifier dengan udara suplai $50^{\circ} \mathrm{C}$ (DB) $20 \% \mathrm{RH}$," Thesis Project, Diponegoro University (2011).

[9] H. D. Young and R. A. Freedman., University Physics, Tenth Edition Translation by Endang Juliastuti., Erlangga 2002. 